\title{
Evaluación in Vitro del Efecto de la Concentración y el Tiempo de Aplicación del Ácido Fluorhídrico sobre la Resistencia a la Flexión Biaxial y la Rugosidad del Disilicato de Litio de Última Generación
}

\author{
In Vitro Evaluation of the Effect of Concentration and Time of Application of \\ Hydrofluoric Acid on the Biaxial Flexural Strength and Roughness of Lithium Disilicate
}

\author{
Carlos Caparroso Pérez ${ }^{*}$; Richard Mejía Bravo**; Juan Felipe Sosa Villa** \& Ángela María Mazo Escobar ${ }^{\star * *}$
}

CAPARROSO, P. C.; MEJíA, B. R.; SOSA, V. J. F. \& MAZO, E. A. M. Evaluación in vitro del efecto de la concentración y el tiempo de aplicación del ácido fluorhídrico sobre la resistencia a la flexión biaxial y la rugosidad del disilicato de litio de última generación. Int. J. Odontostomat., 9(2):273-281, 2015.

RESUMEN: El objetivo fue evaluar in vitro el efecto de la concentración y del tiempo de aplicación del ácido fluorhídrico sobre la rugosidad de la superficie y la resistencia a la fractura de la cerámica de Disilicato de litio de última generación. Se fabricaron 90 discos de cerámica IPS e.max press (14 mm diámetro, 1,2 mm espesor), mediante la técnica de inyección y posterior pulido. Se utilizaron 60 discos para evaluar la resistencia a la flexión biaxial mediante la prueba del pistón y las 3 esferas; se realizó un acondicionamiento con ácido fluorhídrico al $4,6 \%$ y al 9,5\% durante 20,40 y 60 segundos (10 discos por cada tiempo y concentración). Los 30 discos restantes se utilizaron para medir los valores de rugosidad superficial (Ra) con ácido fluorhídrico al $4,6 \%$ y al 9,5\% durante 20, 40 y 60 segundos (5 discos por grupo) mediante microscopia óptica 3D, la cual permitió medir los valores de Rugosidad (Ra). Los datos fueron analizados mediante una prueba de ANOVA y Post- Hoc para determinar las diferencias significativas entre los grupos de estudio. Los valores promedio de la resistencia a la flexión biaxial en los grupos 1, 2 y 3 con

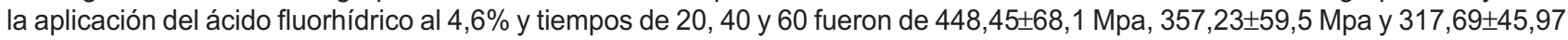

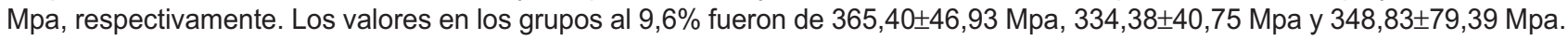
La resistencia a la flexión biaxial de la cerámica de disilicato de litio de última generación se ve afectada por la concentración y el tiempo de aplicación del ácido fluorhídrico. A mayor tiempo de aplicación y concentración del ácido fluorhídrico, menor la resistencia del material. El efecto de la concentración del ácido fluorhídrico y el tiempo de aplicación sobre la cerámica de disilicato de litio no altera significativamente su rugosidad superficial. Según los valores de resistencia a la flexión biaxial encontrados en el presente estudio se recomienda el uso del ácido fluorhídrico a una concentración 4,6\% por 20 segundos tal como lo indica el fabricante.

PALABRAS CLAVE: cerámicas, porcelana dental, disilicato de litio, ácido fluorhídrico, resistencia a la flexión biaxial.

\section{INTRODUCCIÓN}

Las cerámicas dentales son uno de los materiales de elección más utilizados en la actualidad para la restauración de los tejidos dentarios por ofrecer una alta durabilidad, una estética atractiva, resistencia química y una excelente biocompatibilidad (Hooshmand et al., 2008; Esquivel-Upshaw et al., 2008; Etman \& Woolford, 2010). Sus propiedades ópticas permiten imitar la caracterización propia del tejido dentario generando una apariencia más natural; mecánicamente están preparadas para resistir fuerzas compresivas similares o superiores a las toleradas por la dentición natural y las técnicas adhesivas actuales favorecen la longevidad de su restauración en boca (Valenti \& Valenti, 2009).

Odontólogo, especialista en Odontología Integral del Adulto, Magíster en Educación Superior, Profesor asociado, Facultad de Odontología, Universidad de Antioquia, Medellín, Colombia.

* Odontólogo, especialista en Odontología Integral del Adulto, profesor asistente de la Facultad de Odontología, Universidad de Antioquia, Medellín, Colombia.

*** Estudiante de posgrado de odontología Integral del Adulto con énfasis en Prostodoncia. Facultad de Odontología, Universidad de Antioquia, Medellín, Colombia. 
Las investigaciones clásicas, (Malament \& Sokransky, 2001) durante 16 años, demostraron que las restauraciones cementadas con grabado ácido y cemento resinoso tenían una mayor tasa de éxito que las cementadas con oxifosfato sin tratamiento con ácido. Esto se debe a la capacidad de formar uniones fuertes entre la superficie grabada y los cementos resinosos, y hoy en día este concepto ha llevado a que esta técnica sea aplicada casi universalmente (Kelly, 2004).

Para un adecuado funcionamiento de las restauraciones cerámicas vítreas es importante que exista una adhesión entre la cerámica y el sustrato dentario, esta adhesión se puede realizar mediante cementos resinosos que pueden unirse a la estructura dentaria a través de un sistema adhesivo. Sobre el lado de la cerámica, la adhesión es usualmente producida mediante dos mecanismos: por entrecruzamiento micromecánico con el grabado con ácido fluorhídrico o arenado, y mediante adhesión química usando un agente de acople como el silano (Matinlinna et al., 2004). Algunos factores influyen en la resistencia de la adhesión entre la cerámica y la resina compuesta, como la microestructura de la porcelana, el tipo de ácido y el grado de concentración, la composición química del silano, el método de almacenamiento, el agente de cementación y finalmente el método de medición de la resistencia de adhesión (Peumans et al., 2007).

A principios de los 90 s, la técnica de inyección con el método de la cera perdida se introdujo a la odontología como un método innovador para las restauraciones totalmente cerámicas. El sistema IPS Empress (Ivoclar Vivadent), una cerámica vítrea sensible al ácido, reforzada con leucita es uno de los materiales más representativos de las cerámicas prensables. Posteriores mejoras de este sistema llevaron a la introducción del sistema de cerámica vítrea reforzada con disilicato de litio (IPS Empress 2) con una mayor resistencia y más recientemente, reemplazando al Empress 2 apareció el sistema IPS e.max press a base de disilicato de litio con mejores propiedades físicas, mecánicas y translucidez a través de diferentes procesos térmicos (Guess et al., 2010).

Este sistema utiliza el principio de inyección cerámica con pastillas que cuentan con $70 \%$ de cristales de disilicato de litio (Bühler-Zemp \& Völkel, 2005) inmersos en una matriz vítrea que le aportan una resistencia a la flexión de $440 \mathrm{Mpa}$, superando los valores reportados ( $<400 \mathrm{Mpa})$ para el sistema predecesor
(IPS Empress 2) el cual estaba compuesto por un menor porcentaje de cristales de disilicato de litio $(60 \%)$. Esta resistencia a la flexión puede verse afectada por la incorporación de microporosidades en el material al realizar un grabado ácido agresivo (Hooshmand et al.).

El fabricante recomienda para el caso de IPS e.max press un protocolo de acondicionamiento con acido fluorhídrico al $4,6 \%$ por 20 segundos para mejorar las características de adhesión por medio de la disolución de la matriz vítrea y la exposición de los cristales de disilicato de litio generando irregularidades en la superficie de la cerámica (Ivoclar Vivadent Technical, 2005).

Las alteraciones en la superficie por el grabado ácido (Borges et al., 2003), resultan en cambios en el área de superficie y en el comportamiento de humectación de la porcelana. Esto además puede modificar la energía superficial y su potencial adhesivo a la resina. Las diferencias en la composición cerámica producen además cambios topográficos únicos después de los procedimientos de grabado. Así, la microestructura, la composición y los patrones morfológicos de la cerámica después del acondicionamiento de superficie pueden arrojar información potencialmente útil acerca del éxito clínico de los procedimientos adhesivos para este tipo de material (Della Bona, 2005; Dilber et al., 2012).

Actualmente existen en el mercado concentraciones disponibles de ácido fluorhídrico al $4,6 \%$ y al $9,5 \%$ sin encontrarse literatura disponible sobre el efecto que puede causar en la superficie de la cerámica de disilicato de litio de última generación y en su resistencia a la fractura al modificar las concentraciones y los tiempos de grabado ácido recomendados por el fabricante.

El propósito de este estudio es evaluar in vitro el efecto de la concentración y el tiempo de aplicación del ácido fluorhídrico sobre la resistencia a la fractura y la rugosidad de la superficie de la cerámica de disilicato de litio de última generación.

\section{MATERIAL Y MÉTODO}

La preparación de las muestras se llevó a cabo según las especificaciones de la norma ISO 6872 que se refiere a la prueba de resistencia a la flexión biaxial 

resistencia a la flexión biaxial y la rugosidad del disilicato de litio de última generación. Int. J. Odontostomat., 9(2):273-281, 2015.

de las cerámicas dentales. Se determinó que el tamaño de la muestra fuera de 90 discos mediante un muestreo por conveniencia.

Se fabricaron 90 discos en cera con un diámetro de $14 \mathrm{~mm}$ por $1,4 \mathrm{~mm}$ de espesor, los cuales se unieron a un bebedero de cera prefabricado de 2,5 $\mathrm{mm}$ de diámetro (Fig. 1). Se revistieron en 100 gramos de material de revestimiento IPS Press Vest Speed (Ivoclar Vivadent ${ }^{\mathrm{TM}}$, Schaan, Liechtenstein) mezclados con $16 \mathrm{ml}$ de líquido de revestimiento y 11 $\mathrm{ml}$ de agua destilada al vacio con el equipo EasyMix de Bego por 90 segundos. Posteriormente se llevó el material de revestimiento por 45 minutos al horno Radiance multi-stage MSM de Jelrus precalentado a $850^{\circ} \mathrm{C}$ para una adecuada evaporación de la cera. El anillo de inyección se llevó al horno de prensado

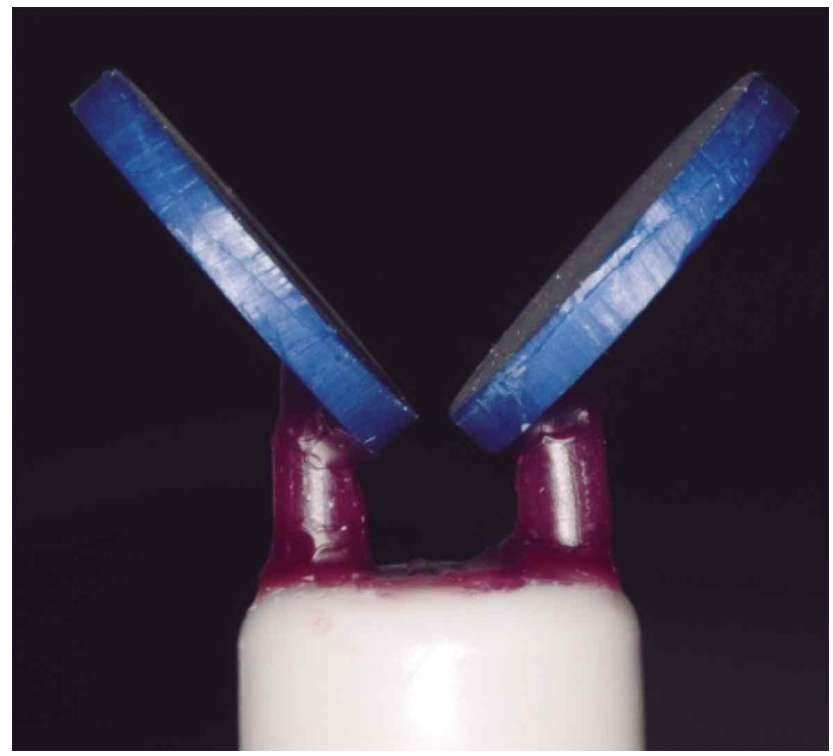

Fig. 1. Ubicación de bebederos Renfert en los discos de cera para revestirlos en los anillos de $100 \mathrm{~g}$ de Emax Press Speed investment, Ivoclar VivadentTM.

Tabla I. Resistencia a la flexión biaxial.

\begin{tabular}{lcc}
\hline \multirow{2}{*}{ Tiempo } & \multicolumn{2}{c}{ Concentración } \\
\cline { 2 - 3 } 20 segundos (control) & $\mathbf{4 , 6 \%}$ & $\mathbf{9 , 5 \%}$ \\
40 segundos & 10 discos & 10 discos \\
60 segundos & 10 discos & 10 discos \\
\hline
\end{tabular}

Tabla II. Rugosidad superficial.

\begin{tabular}{lcc}
\hline & \multicolumn{2}{c}{ Concentración } \\
\cline { 2 - 3 } Tiempo & $\mathbf{4 , 6 \%}$ & $\mathbf{9 , 5 \%}$ \\
\hline 20 segundos (control) & 5 discos & 5 discos \\
40 segundos & 5 discos & 5 discos \\
60 segundos & 5 discos & 5 discos \\
\hline
\end{tabular}

Programat EP5000 (Ivoclar Vivadent; Schaan, Liechtenstein) a una temperatura de $915^{\circ} \mathrm{C}$ para la inyección de las pastillas de cerámica de disilicato de litio E.max press LT A2. El anillo inyectado se dejó a temperatura ambiente por 30 minutos aproximadamente para su enfriamiento.

La remoción del revestimiento sobre los disco cerámicos se realizó mediante arenado con partículas de óxido de aluminio de $110 \mu \mathrm{m}$ a dos bares de presión atmosférica (30 psi) a una distancia de $10 \mathrm{~mm}$ de la muestra en un arenador Duostar de Bego.

Los discos se pulieron con papel de lija de agua calibre 400, 600 y 1200 sucesivamente hasta dejar la superficie homogénea y lisa; luego fueron lavados con ultrasonido por cinco minutos. Las medidas finales de los discos fueron de $14 \mathrm{~mm}$ de diámetro x 1,2 mm de espesor, las cuales se estandarizaron mediante un calibrador digital.

Para evaluar la resistencia a la flexión biaxial se utilizaron 60 discos cerámicos seleccionados aleatoriamente y divididos en 6 grupos de 10 discos cada grupo (Tabla I).

Para evaluar la rugosidad superficial se utilizaran 30 discos cerámicos seleccionados aleatoriamente y divididos en 6 grupos de 5 discos cada grupo (Tabla II).

Se tomaron los discos de porcelana de cada grupo de investigación y se grabaron en la superficie con ácido fluorhídrico IPS Ceramic Etching Gel ${ }^{\circledR}$ (Ivoclar Vivadent; Schaan, Liechtenstein) al 4,6\% y ácido fluorhídrico Eufar® al 9,5\% por 20, 40 y 60 segundos respectivamente (Figs. 2, 3 y 4). Posteriormente se lavaron con abundante agua durante 30 segundos y se secaron al aire.

La resistencia a la flexión biaxial (Della Bona, 2005) se determinó mediante la prueba del pistón y 3 esferas en 10 muestras de cada grupo según la concentración del ácido y el tiempo. Los discos se ubicaron en el centro del soporte que consiste en 3 esferas de acero ( $3,18 \mathrm{~mm}$ de diámetro) ubicadas cada una a $6 \mathrm{~mm}$ del centro del soporte. Entre el pistón y el disco se colocó una hoja de polietileno de espesor delgado $(0,05 \mathrm{~mm})$ para facilitar la distribución de las cargas de manera uniforme. La carga se aplicó al centro de cada muestra mediante un cilindro plano (pistón) perpendicular a la superficie. Las muestras se cargaron en una maquina de prueba Universal Instron ${ }^{\mathrm{TM}}$, modelo 2519-105 con capacidad de carga de 500 $\pm 0,025$ 


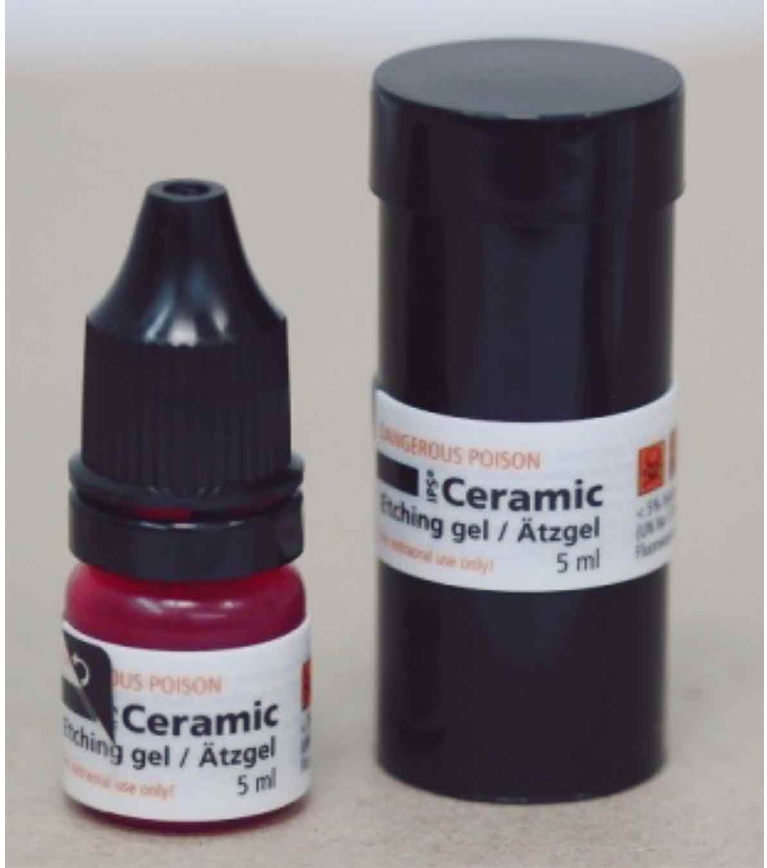

Fig. 2. Ácido fluorhídrico IPS Ceramic Etching Gel® al $4,6 \%$.

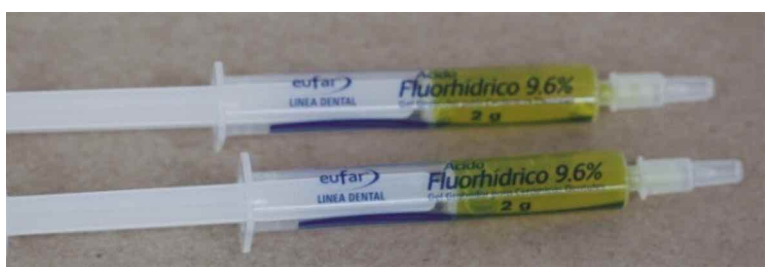

Fig. 3. Ácido fluorhídrico Eufar® al 9,6\%.

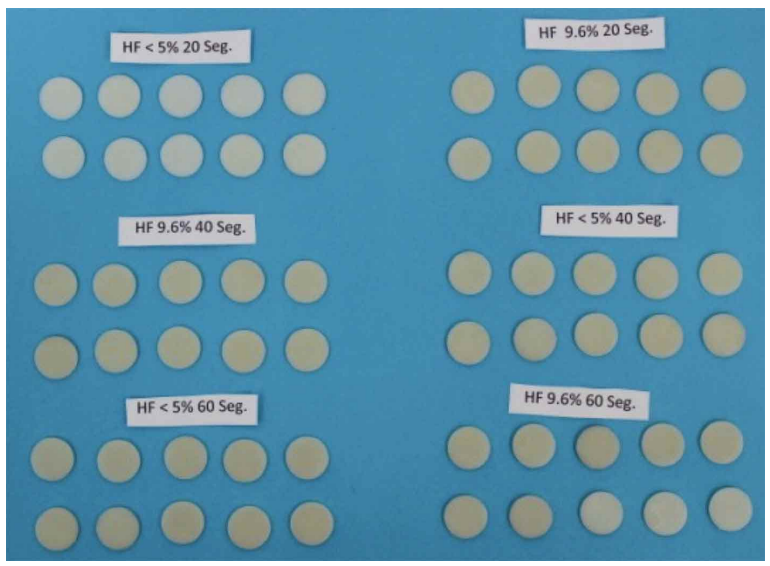

Fig. 4. Grupos de estudio según tiempo y concentración.

$\mathrm{N}$ a una velocidad de $0,5 \mathrm{~mm} / \mathrm{min}$ hasta la fractura (Figs. 5 y 6). Los valores de máxima fuerza (MPa) que corresponden a la resistencia a la flexión biaxial se calcularon de acuerdo a la siguiente ecuación sugerida por la prueba estándar (NSAI Standards, 2015):

$$
S=-0.2387 P(X-Y) / d^{2}
$$

Donde $S$ es la máxima concentración de fuerza (MPa), $\mathrm{P}$ es la carga a la que se produce la fractura (Newtons) y d es el espesor de la muestra en milímetros en el origen de la fractura.

$X$ y $Y$ se determinaron de la siguiente manera:

$X=(1+v) \ln (B / C) 2+[(1-n) / 2](B / C) 2$,

$Y=(1+n)[1+\ln (A / C) 2]+(1-v)(A / C) 2$,

Donde $\mathrm{n}$ es el coeficiente de Poisson, A es el radio del circulo de soporte $(\mathrm{mm})$, B es el radio de la punta del pistón $(\mathrm{mm})$ y C es el radio de la muestra $(\mathrm{mm})$. Los valores del coeficiente de Poisson para el disilicato de litio se asumen como 0,23 (Hooshmand).

La rugosimetría en cada uno de los 5 discos perteneciente a cada grupo de estudio se determinó mediante un microscopio óptico NIKON eclipce Iv1000 con un software $\mathrm{NI}$ Elements 3.1 el cual permitió la reconstrucción tridimensional de toda la superficie analizada y de un perfil especifico a un aumento de 20X para describir posteriormente el valor promedio de la rugosidad $(\mathrm{Ra})$.

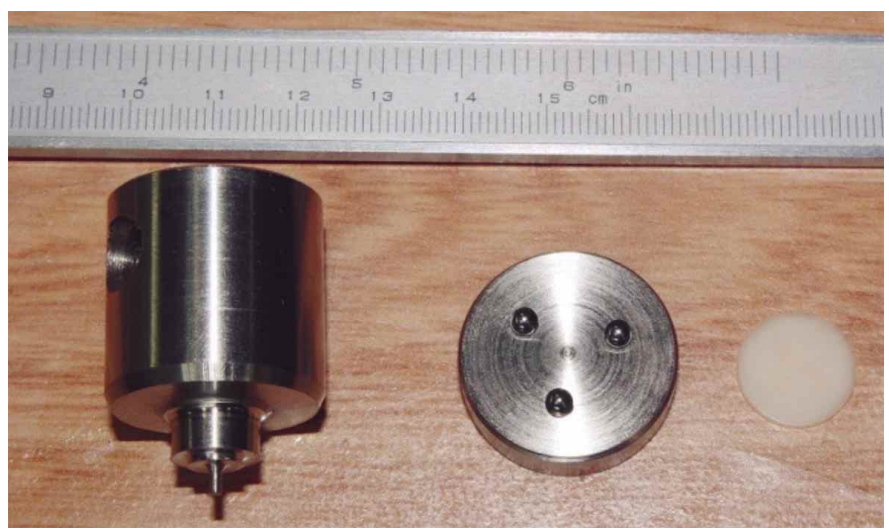

Fig. 5. Dispositivos usados en la prueba de flexión biaxial.

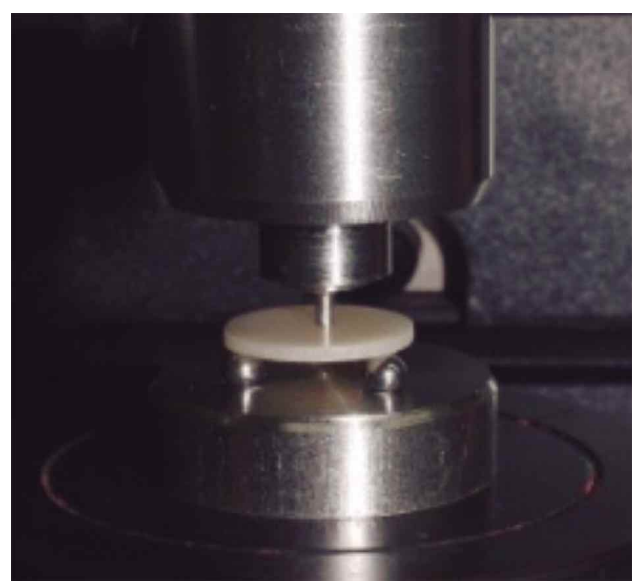

Fig. 6. Prueba de flexión biaxial aplicada al centro de cada muestra mediante un cilindro plano (pistón) perpendicular a la superficie en una maquina de prueba Universal Instron ${ }^{\mathrm{TM}}$. 
El análisis estadístico de los datos se realizó con el programa SPSS $®$ versión 19. Los datos se expresaron en promedio con su respectiva desviación estándar (variables con distribución normal según la prueba de Kolmogorov-Smirnov). Se realizaron comparaciones aplicando un análisis de varianza de una vía, cuando se encontraron diferencias se utilizó el análisis de Post Hoc con la prueba Bonferroni y se consideró una significancia estadística cuando el valor de $\mathrm{P}$ fue menor a 0,05.

\section{RESULTADOS}

Resistencia a la flexión biaxial. Los valores promedio de la resistencia a la flexión biaxial en los grupos 1,2 y 3 con la aplicación del HFL al 4,6\% y tiempos de 20,40 y 60 fueron de $448,45 \pm 68,1 \mathrm{Mpa}, 357,23 \pm 59,5$ Mpa y $317,69 \pm 45,97 \mathrm{Mpa}$, respectivamente. Los valores en los grupos con la aplicación al 9,6\% fueron de $365,40 \pm 46,93 \mathrm{Mpa}, 334,38 \pm 40,75$ Mpa y $348,83 \pm 79,39$ Mpa (Tabla III).

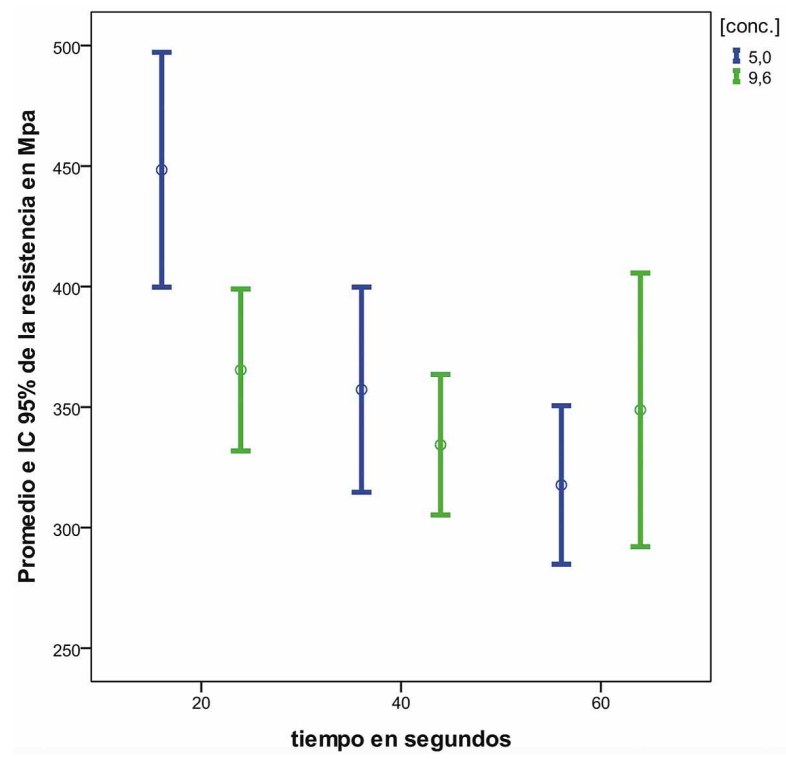

Fig. 7. El efecto de la concentración del ácido fluorhídrico sobre la resistencia a la flexión biaxial sobre la cerámica de disilicato de litio de última generación a los 20, 40 y 60 s.
El efecto de la concentración no mostró diferencias significativas en la resistencia a la flexión biaxial $(P>0.05)$. Al contrario, el tiempo de aplicación del ácido fluorhídrico sí fue un factor determinante en la resistencia a la flexión biaxial de la cerámica de disilicato de litio de última generación; Valor $\mathrm{P}=0,001$ para la prueba de ANOVA con un promedio de resistencia de $448,45 \pm 68,1 \mathrm{MPa}$ mucho mayor a los 20 segundos de aplicación del ácido (Fig. 7).

Al comparar la resistencia a la flexión biaxial de la cerámica de disilicato de litio a los 20 segundos de exposición al acido fluorhídrico a una concentración $4,6 \%$ y del $9,6 \%$ se observaron diferencias estadísticamente significativas, mientras que al comparar la resistencia de la cerámica a la flexión biaxial a los 40 segundos de exposición al acido fluorhídrico a una concentración $4,6 \%$ y del $9,6 \%$ no se observaron diferencias estadísticamente significativas y la misma tendencia se observó para el grupo de exposición de la cerámica de disilicato de litio a 60 segundos a las distintas concentraciones.

Teniendo en cuenta la resistencia a la flexión biaxial aplicando el ácido fluorhídrico a una concentración $4,6 \%$ por los distintos tiempos, se observaron diferencias significativas en la aplicación a los 20 y 40 segundos y a los 20 y 60 segundos siendo más resistente las cerámicas cuando fueron expuestas al acido por 20 segundos. No se observaron diferencias estadísticamente significativas en la flexión biaxial entre los 40 y 60 segundos de aplicación del ácido. Al comparar la resistencia a la flexión biaxial con una aplicación del ácido a una concentración del 9,6\% entre los diferentes tiempos de exposición del acido no se encontraron diferencias.

Rugosidad de la superficie grabada . Los valores promedio de la rugosidad superficial de la cerámica de disilicato de litio grabada con ácido fluorhídrico en concentraciones $4,6 \%$ y al $9,6 \%$ con su desviación estándar para los grupos de estudio, así como su análisis estadístico, se presentan en la Tabla IV.

Tabla III. Resistencia a la flexión biaxial de la cerámica de disilicato de litio.

\begin{tabular}{lccccc}
\hline & & \multicolumn{3}{c}{ Tiempo } \\
\cline { 2 - 6 } Concentración & $\mathbf{2 0}$ segundos & $\mathbf{4 0}$ segundos & $\mathbf{6 0}$ segundos & Valor P & Total (en Mpa) \\
\hline $4,6 \%$ & $448,45 \pm 68,1^{\mathrm{a}}$ & $357,23 \pm 59,5^{\mathrm{b}}$ & $317,69 \pm 45,9 \mathrm{~b}$ & 0,00 & $374,46 \pm 79,34$ \\
$9,6 \%$ & $365,40 \pm 46,9^{\mathrm{b}}$ & $334,38 \pm 40,7^{\mathrm{b}}$ & $348,83 \pm 79,3 \mathrm{~b}$ & 0,50 & $349,54 \pm 57,63$ \\
Valor $\mathrm{P}$ & 0,005 & 0,330 & 0,297 & -- & 0,169 \\
Total & $406,92 \pm 71,0^{\mathrm{a}}$ & $345,80 \pm 50,9^{\mathrm{b}}$ & $333,26 \pm 65,1 \mathrm{~b}$ & 0,001 & --- \\
\hline
\end{tabular}

Las letras en superíndice coincidentes no muestran diferencias significativas, mientras que las letras en superíndice discordantes si muestran diferencias entre los tiempos de aplicación del acido. 
Tabla IV. Rugosidad promedio en micras del disilicato de litio posterior al acondicionamiento con ácido fluorhídrico.

\begin{tabular}{lccccc}
\hline & \multicolumn{5}{c}{ Tiempo } \\
\cline { 2 - 6 } Concentración & $\mathbf{2 0}$ segundos & $\mathbf{4 0}$ segundos & $\mathbf{6 0}$ segundos & Valor $\mathbf{P}$ & Total (en Ra) \\
\hline$<5 \%$ & $5,647 \pm 0,48$ & $6,297 \pm 1,61$ & $6,298 \pm 0,90$ & 0,575 & $6,08 \pm 1,06$ \\
$9,6 \%$ & $5,863 \pm 1,81$ & $5,211 \pm 0,91$ & $6,515 \pm 0,76$ & 0,296 & $5,86 \pm 1,28$ \\
Valor $\mathrm{P}$ & 0,805 & 0,226 & 0,694 & -- & 0,618 \\
Total & $5,75 \pm 1,26$ & $5,75 \pm 1,35$ & $6,40 \pm 0,80$ & 0,366 & $5,97 \pm 1,16$ \\
\hline
\end{tabular}

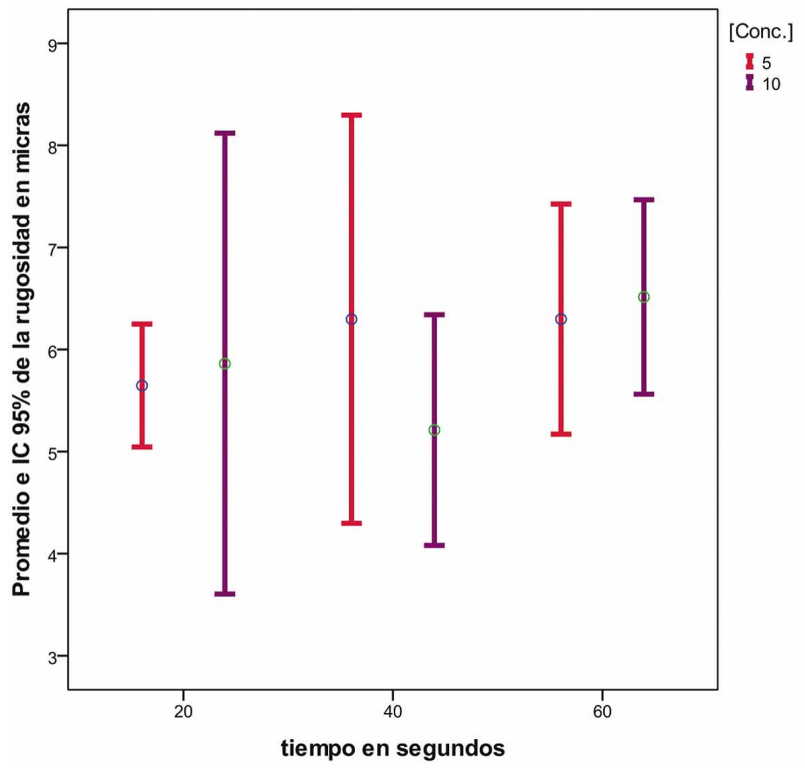

Fig. 8. El efecto de la concentración del ácido fluorhídrico sobre las características de la rugosidad superficial de la cerámica de disilicato de litio de última generación a los 20 , 40 y $60 \mathrm{~s}$.

El efecto de la concentración del ácido fluorhídrico no mostró diferencias significativas en las características de la rugosidad superficial de la cerámica $(P=0,618)$. De igual manera, al evaluar el efecto del tiempo de aplicación del ácido fluorhídrico no se encontraron diferencias estadísticamente significativas con respecto a la rugosidad superficial de la cerámica de disilicato de litio $(P=0,366)$ (Tabla IV, Fig. 8).

\section{DISCUSIÓN}

La resistencia mecánica es un factor importante que controla el éxito clínico de las restauraciones dentales. En los materiales frágiles como la porcelana, se mide la resistencia a la compresión por conveniencia ya que estos materiales son más resistentes a estas fuerzas que a las de tracción, por lo tanto es más significativo medir la resistencia a la tracción en las cerámicas al evaluar una posible falla de la restauración dental, especialmente en presencia de defectos de superficie (Ban \& Anusavice, 1990).

Para determinar la resistencia a la tracción de los materiales dentales frágiles se debería utilizar una prueba de compresión diametral pero no está indicado por las dificultades que representa la alineación de la muestra durante la prueba y además lleva a distintos patrones de fractura lo cual no puede ser considerado muy confiable, por esto se ha utilizado la prueba de flexión biaxial que tiene como ventaja que la tensión es generada en un solo punto de la estructura (Ban \& Anusavice).

La prueba de flexión biaxial se ha utilizado con frecuencia para la determinación de las características de la fractura de materiales frágiles. La medición de la resistencia de los materiales frágiles bajo flexión biaxial en lugar de flexión uniaxial a menudo se considera más confiable, ya que la tensión máxima se produce en la zona central. Esta característica hace que sea el método apropiado para evaluar los efectos de las condiciones de superficie en la resistencia (Ban \& Anusavice).

Para el presente estudio el valor de la resistencia a la flexión biaxial del sistema cerámico IPS e.max press a base de disilicato de litio manejado según las instrucciones del fabricante fue de $448,45 \pm 68,10 \mathrm{MPa}$, lo cual está de acuerdo con los $440 \mathrm{MPa}$, valor reportado por el estudio de Albakry et al. (2003), para el sistema IPS e.max Press.

Sin embargo, los resultados del presente estudio superan los valores encontrados por Guazzato et al. (2004) de $340 \mathrm{MPa}$ para el sistema Empress 2 y por Chen et al. (2008), quienes reportaron un valor de $355 \mathrm{MPa}$ para este mismo sistema, lo cual está de acuerdo con las propiedades mecánicas de la microestructura de sistema IPS Empress 2 que presenta un menor porcentaje de cristales de disilicato de litio. 
El proceso de cementación es vital para el éxito clínico de las restauraciones cerámicas; en el sistema IPS e.max® Press el protocolo recomendado por el fabricante es de un acondicionamiento con ácido fluorhídrico al 4,6\% por 20 segundos (Della Bona, 2005) para generar una adecuada adhesión entre el agente cementante y la restauración cerámica, incrementando las irregularidades en la superficie del material que permiten una mejor adhesión, pero modifican sus propiedades mecánicas. Tal como lo confirma el presente estudio, tanto la concentración del ácido fluorhídrico como el tiempo de aplicación afectan la resistencia a la flexión de la cerámica de disilicato de litio; que en tanto si se aumentan el tiempo como la concentración, la resistencia a la flexión biaxial disminuye. Confirmando que se deben seguir las recomendaciones del fabricante ya que al aplicar ese protocolo se observó la mayor resistencia a la flexión biaxial.

Lo anterior está de acuerdo con el estudio de Hooshmand et al., sobre el efecto del grabado con ácido fluorhídrico al $9 \%$ por dos minutos sobre la superficie de las cerámicas prensables a base de disilicato de litio y a base de leucita (sistema IPS. Empress 2 y IPS Empress) en donde se observó una disminución significativa en la resistencia a la flexión posterior, a la prueba de flexión biaxial, reportando un valor de resistencia de 250,56 MPa para el disilicato de litio; con lo cual los autores concluyen que el grabado ácido puede disminuir la resistencia de las cerámicas prensables.

El estudio actual concuerda con los resultados obtenidos por Zogheib et al. (2011) en el que evaluaron el efecto de diferentes periodos de tiempo de grabado ácido (20,60, 90 y 180 segundos) sobre la rugosidad superficial y la resistencia a la flexión de una cerámica de disilicato de litio; ellos encontraron que el aumento en el periodo de tiempo de grabado ácido de la cerámica con ácido fluorhídrico, afectó significativamente $(p=0,003)$ la rugosidad superficial y la resistencia a la flexión de la cerámica de disilicato de litio.

Las superficies rugosas se desgastan más rápidamente y tienen un mayor coeficiente de fricción que las superficies lisas. La rugosidad es un buen parámetro para estimar el desempeño de un componente mecánico, ya que sus irregularidades en la superficie pueden formar lugares de nucleación para las grietas y corrosión. Existen diversos parámetros de rugosidad usados, aunque el $\mathrm{Ra}$ (promedio aritmético de la rugosidad de la superficie) es el más usado.
Debido a que esos parámetros reducen toda la información perfilométrica a números (micras), se debe tener mucho cuidado en la aplicación e interpretación de dichos valores (Della Bona, 2009).

En el presente estudio los valores promedio de la rugosidad en la cerámica de disilicato de litio acondicionada con ácido fluorhídrico en concentraciones del $4,6 \%$ y $9,5 \%$ por 20,40 y 60 segundos fueron superiores a los valores encontrados por Zogheib et al. Las diferencias entre estos dos estudios puede deberse a la técnica de fabricación de cada una de las muestras por analizar. En el estudio de Zogheib et al., se fabricaron mediante un sistema de corte sobre la cerámica lo que puede generar una superficie más lisa y homogénea; mientras las muestras del presente estudio fueron pulidas manualmente mediante el uso de diferentes tamaños de grano (desde el más grueso al más fino) hasta finalizar en un pulido de espejo, pudiendo mantener en su superficie imperfecciones mayores para este sistema de pulido. Además se observa que el estudio de Zogheib et al., analizaron secciones o áreas de la muestra mucho más pequeñas utilizando un sistema diferente de observación como la microscopía electrónica de barrido (SEM) con un aumento mucho mayor; mientras que el presente estudio utiliza microscopía óptica sobre áreas grabadas mayores con un menor aumento. Sin embargo, ambos estudios coinciden al observar la misma tendencia a aumentar el grado de rugosidad debido a la generación de poros y canales de mayor tamaño a medida que aumenta el periodo de tiempo del grabado ácido con ácido fluorhídrico.

A partir de las limitaciones del presente estudio se sugiere la evaluación de las propiedades mecánicas de la cerámica de disilicato de litio e.max press con tiempos de grabado ácido menores para la concentración del 9,6\% del ácido fluorhídrico, lo cual pueda generar un protocolo que mantenga las propiedades físicas y mecánicas del material cerámico.

\section{CONCLUSIONES}

La resistencia a la flexión biaxial de la cerámica de disilicato de litio de última generación se ve afectada por la concentración y el tiempo de aplicación del ácido fluorhídrico. A mayor tiempo de aplicación y concentración del ácido fluorhídrico, menor la resistencia del material. 

resistencia a la flexión biaxial y la rugosidad del disilicato de litio de última generación. Int. J. Odontostomat., 9(2):273-281, 2015.

El efecto de la concentración del ácido fluorhídrico y el tiempo de aplicación sobre la cerámica de disilicato de litio no altera significativamente la rugosidad de la superficie cerámica.
Según los valores de resistencia a la flexión biaxial encontrados en el presente estudio se recomienda el uso del ácido fluorhídrico a una concentración $4,6 \%$ por 20 segundos tal como lo indica el fabricante.

CAPARROSO, P. C.; MEJÍA, B.R.; SOSA, V. J. F. \& MAZO, E. Á. M. In vitro evaluation of the effect of concentration and time of application of hydrofluoric acid on the biaxial flexural strength and roughness of lithium disilicate. Int. J. Odontostomat., 9(2):273-281, 2015.

ABSTRACT: The purpose of this study was to evaluate in vitro the effect of concentration and time of application of hydrofluoric acid on the surface roughness and fracture resistance of lithium disilicate ceramic generation. 90 IPS e.max press ceramic discs ( $14 \mathrm{~mm}$ diameter, $1.2 \mathrm{~mm}$ thick) were fabricated by the press technique and then polished. 60 discs to evaluate the biaxial flexural strength test by the piston and the three spheres were used; under $4.6 \%$ hydrofluoric acid and $9.6 \%$ conditioned treatment was carried out for 20,40 and 60 seconds (10 disks per each time and concentration). The remaining 30 discs were used to measure the surface roughness values (Ra) with $4.6 \%$ and $9.6 \%$ hydrofluoric acid for 20 , 40 and 60 seconds (5 discs per group) using 3D optical microscopy, which it possible to measure the values of roughness (Ra). Data were analyzed by ANOVA and Post Hoc to determine significant differences between the study groups. Average values of the biaxial flexural strength in groups 1, 2 and 3 with the application of $4.6 \% \mathrm{HFL}$ and times of 20,40 and 60 seconds were $448.45 \pm 68.1 \mathrm{MPa}, 357.23 \pm 59.5 \mathrm{MPa}$ and $317.69 \pm 45.97 \mathrm{MPa}$ respectively. The values in groups of $9.6 \%$ were $365.40 \pm 46.93 \mathrm{MPa}, 334.38 \pm 40.75$ and $348.83 \pm 79.39 \mathrm{Mpa}$. The biaxial flexural strength of the ceramic lithium disilicate generation is affected by the concentration and time of application of hydrofluoric acid. A longer time of application and concentration of hydrofluoric acid, less material strength. The effect of the concentration of hydrofluoric acid and application time on the ceramic lithium disilicate did not significantly alter the roughness of the ceramic surface. According to the values of biaxial flexural strength found in the present study the use of hydrofluoric acid is recommended at a concentration $4.6 \%$ for 20 seconds as indicated by the manufacturer.

KEY WORDS: ceramics, dental porcelain, lithium disilicate, hydrofluoric acid, biaxial flexural strength.

\section{REFERENCIAS BIBLIOGRÁFICAS}

Albakry, M.; Guazzato, M. \& Swain, M. V. Biaxial flexural strength, elastic moduli, and x-ray diffraction characterization of three pressable all-ceramic materials. J. Prosthet. Dent., 89(4):374-80, 2003.

Ban, S. \& Anusavice, K. J. Influence of test method on failure stress of brittle dental materials. J. Dent. Res., 69(12):1791-9, 1990.

Borges, G. A.; Sophr, A. M.; de Goes, M. F.; Sobrinho, L. C. \& Chan, D. C. Effect of etching and airborne particle abrasion on the microstructure of different dental ceramics. J. Prosthet. Dent., 89(5):479-88, 2003.

Bühler-Zemp, P. \& Völkel, T. Documentación Científica IPS e.max® ZirCAD. Schaan, Ivoclar Vivadent AG, 2005.

Chen, Y. M.; Smales, R. J.; Yip, K. H. \& Sung, W. J. Translucency and biaxial flexural strength of four ceramic core materials. Dent. Mater., 24(11):1506-11, 2008.

Della Bona, A. Characterizing ceramics and the interfacial adhesion to resin: II- the relationship of surface treatment, bond strength, interfacial toughness and fractography. J. Appl. Oral Sci., 13(2):101-9, 2005.
Della Bona, A. Principios de adhesión aplicados a las cerámicas odontológicas. En: Della Bona A. Adhesion a las cerámicas. Evidencias científicas para el uso clínico. Sao Paulo, Editora Artes Medicas Ltda., 2009. pp.91-132.

Dilber, E.; Yavuz, T.; Kara, H. B. \& Ozturk, A. N. Comparison of the effects of surface treatments on roughness of two ceramic systems. Photomed. Laser Surg., 30(6):308-14, 2012.

Esquivel-Upshaw, J. F.; Young, H.; Jones, J.; Yang M. \& Anusavice, K. J. Four-year clinical performance of a lithia disilicate-based core ceramic for posterior fixed partial dentures. Int. J. Prosthodont., 21(2):155-60, 2008.

Etman, M. K. \& Woolford, M. J. Three-year clinical evaluation of two ceramic crown systems: a preliminary study. J. Prosthet. Dent., 103(2):80-90, 2010.

Guazzato, M.; Albakry, M.; Ringer, S. P. \& Swain, M. V. Strength, fracture toughness and microstructure of a selection of all-ceramic materials. Part I. Pressable and alumina glass-infiltrated ceramics. Dent. Mater., 20(5):441-8, 2004. 
CAPARRoso, P. C.; MEJÍA, B. R.; SOSA, V. J. F. \& MAZO, E. A. M. Evaluación in vitro del efecto de la concentración y el tiempo de aplicación del ácido fluorhídrico sobre la resistencia a la flexión biaxial y la rugosidad del disilicato de litio de última generación. Int. J. Odontostomat., 9(2):273-281, 2015.

Guess, P. C.; Zavanelli, R. A.; Silva, N. R.; Bonfante, E. A.; Coelho, P. G. \& Thompson, V. P. Monolithic CAD/CAM lithium disilicate versus veneered Y-TZP crowns: comparison of failure modes and reliability after fatigue. Int. J. Prosthodont., 23(5):434-42, 2010.

Hooshmand, T.; Parvizi, S. \& Keshvad, A. Effect of surface acid etching on the biaxial flexural strength of two hotpressed glass ceramics. J. Prosthodont., 17(5):415-9, 2008.

Ivoclar Vivadent Technical. Instrucciones de Uso IPS e.max® Press. Schaan, Ivoclar Vivadent AG, 2005.

Kelly, J. R. Dental ceramics: current thinking and trends. Dent. Clin. North Am., 48(2):513-30, 2004.

Malament, K. A. \& Socransky, S. S. Survival of Dicor glassceramic dental restorations over 16 years. Part III: effect of luting agent and tooth or tooth-substitute core structure. J. Prosthet. Dent., 86(5):511-9, 2001.

Matinlinna, J. P.; Lassila, L. V.; Ozcan, M.; Yli-Urpo, A. \& Vallittu, P. K. An introduction to silanes and their clinical applications in dentistry. Int. J. Prosthodont., 17(2):15564, 2004.

NSAI Standards. Dentistry - Ceramic materials (ISO 6872:2015). Ginebra, International Standard Organization, 2015.

Peumans, M.; Hikita, K.; De Munck, J.; Van Landuyt, K.; Poitevin, A.; Lambrechts, P. \& Van Meerbeek, B. Effects of ceramic surface treatments on the bond strength of an adhesive luting agent to CAD-CAM ceramic. J. Dent., 35(4):282-8, 2007.

Valenti, M. \& Valenti, A. Retrospective survival analysis of 261 lithium disilicate crowns in a private general practice. Quintessence Int., 40(7):573-9, 2009.

Zogheib, L. V.; Bona, A. D.; Kimpara, E. T. \& MacCabe, J. F. Effect of hydrofluoric acid etching duration on the roughness and flexural strength of a lithium disilicatebased glass ceramic. Braz. Dent. J., 22(1):45-50, 2011.
Dirección para Correspondencia:

Carlos Caparroso

Odontólogo, especialista en Odontología Integral del Adulto

Magíster en Educación Superior

Profesor Asociado

Facultad de Odontología

Universidad de Antioquia

Antioquia

COLOMBIA

Email: ccaparroso@gmail.com

Recibido: 01-03-2015

Aceptado: 10-07-2015 